\title{
ON POLYNOMIAL IDENTITIES FOR RECURSIVE SEQUENCES
}

\author{
I. MARTINJAK AND I. VRSALJKO
}

Received 09 September, 2015

\begin{abstract}
In this paper we extend the notion of Melham sum to the Pell and Pell-Lucas sequences. While the proofs of general statements rely on the binomial theorem, we prove some spacial cases by the known Pell identities. We also give extensions of obtained expressions to the other recursive sequences.
\end{abstract}

2010 Mathematics Subject Classification: 34B10; 34B15

Keywords: Pell sequence, Pell-Lucas sequence, Pell identities

\section{INTRODUCTION}

The Pell sequence $\left(P_{n}\right)_{n \geq 0}$ and the Pell-Lucas sequence $\left(Q_{n}\right)_{n \geq 0}$ are defined as the second order recurrences,

$$
\begin{aligned}
& P_{n+2}=2 P_{n+1}+P_{n}, \quad P_{0}=0, \quad P_{1}=1 \\
& Q_{n+2}=2 Q_{n+1}+Q_{n}, \quad Q_{0}=2, \quad Q_{1}=2 \text {. }
\end{aligned}
$$

Equivalently, these sequences can be defined as the solutions of Diophantine equations

$$
x^{2}-d y^{2}= \pm 1
$$

for $d=2$. More precisely, the pairs $\left(Q_{n} / 2, P_{n}\right)$ are all solutions of these equations. The $n$-th term of the Pell sequence can also be expressed by the closed form equation. The Pell-Lucas sequence is sometimes called companion Pell sequence and there is also similar closed form for this sequence. We let $\gamma$ denote the silver ratio, $\gamma:=$ $1+\sqrt{2}$ and we set $\delta:=1-\sqrt{2}$. Then the closed formula for Pell sequence can be written as

$$
P_{n}=\frac{\gamma^{n}-\delta^{n}}{\gamma-\delta}
$$

while for the companion Pell numbers we have $Q_{n}=\gamma^{n}-\delta^{n}$.

Both the Pell sequence and Pell equation are the subject of numerous papers. Among the most remarkable theoretical number properties let us mention the facts that

- $P_{2 n+1}$ divides the sum $\sum_{k=0}^{2 n} P_{2 k+1}$, 
- $P_{2 n}$ divides the sum $\sum_{k=1}^{2 n} P_{2 k-1}$,

and sum of the first $4 n+1$ Pell numbers $\sum_{k=1}^{4 n+1} P_{k}$ is a perfect square [7]. Furthermore, in [6] Dujella found quadruples of the Pell and Pell-Lucas numbers that have the property of Diophantus of a certain order. In particular, the quadruples

$$
\begin{gathered}
\left\{P_{2 n}, P_{2 n+2}, 2 P_{2 n}, Q_{2 n} P_{2 n+1} Q_{2 n+1}\right\}, \\
\left\{P_{2 n}, P_{2 n+2}, 2 P_{2 n+2}, P_{2 n+1} Q_{2 n+1} Q_{2 n+2}\right\}
\end{gathered}
$$

have the property of Diophantus of order 1 , meaning that $a_{i} a_{j}+1$, is a perfect square where $a_{i}, a_{j}, i \neq j$ are the elements of a quadruple.

Some recent surveys on the Pell equation one can find in [1,3].

There are also many known combinatorial properties and identities for Pell and Pell-Lucas sequences $[4,5,12]$. This includes several identities encountering both of the sequences,

$$
Q_{n}=P_{n-1}+P_{n+1}
$$

being the basic one. Recall that the Cassini identity [11] for Pell numbers has form

$$
P_{n-1} P_{n+1}-P_{n}^{2}=(-1)^{n} .
$$

An elegant proof is based on the fact that

$$
\left(\begin{array}{ll}
0 & 1 \\
1 & 2
\end{array}\right)^{n}=\left(\begin{array}{cc}
P_{n-1} & P_{n} \\
P_{n} & P_{n+1}
\end{array}\right)
$$

which can be proved by induction. When applying the Cauchy-Binet theorem for determinants, the statement follows immediately. We will also use relation

$$
P_{m+n}=P_{m-1} P_{n}+P_{m} P_{n+1},
$$

for the purpose to prove some polynomial identities for Pell numbers. Identity (1.6) can be proved by induction.

This paper aims at finding Pell identities and polynomial representation for the Pell numbers. In what follows, firstly we prove that $(2 m+1) n$-th Pell number is represented as a polynomial in $P_{n}$. Then we extend the notion of Melham sum [10] to the Pell and Pell-Lucas sequences and find related expansions into the power series of $P_{n}$, where exponents are odd. Finally, we give extensions of the obtained identities for a certain, more general class of recursive sequences.

\section{The $(2 m+1) n$-TH Pell NUMber as A POLYNOMial IN $P_{n}$}

Proposition 1. For the Pell sequence $\left(P_{n}\right)_{n \geq 0}$ we have

$$
\begin{aligned}
& \text { i) } \quad P_{3 n}=8 P_{n}^{3}+3(-1)^{n} P_{n} \\
& \text { ii) } \quad P_{5 n}=64 P_{n}^{5}+40(-1)^{n} P_{n}^{3}+5 P_{n} \text {. }
\end{aligned}
$$


Proof. According to relations (1.5) and (1.6) we get

$$
\begin{aligned}
P_{3 n} & =P_{2 n+n}=P_{2 n-1} P_{n}+P_{2 n} P_{n+1} \\
& =P_{n-1}^{2} P_{n}+P_{n}^{3}+\left(P_{n}-1 P_{n}+P_{n} P_{n+1}\right)\left(2 P_{n}+P_{n-1}\right) \\
& =P_{n}\left(P_{n-1}^{2}+2 P_{n} P_{n-1}+P_{n}^{2}+2 P_{n} P_{n+1}+P_{n-1}^{2}+P_{n}^{2}+(-1)^{n}\right)
\end{aligned}
$$

which finally gives

$$
\begin{aligned}
P_{3 n} & =P_{n}\left(3 P_{n}^{2}+2(-1)^{2}+2 P_{n} P_{n+1}+P_{n-1}^{2}\right) \\
& =P_{n}\left(3 P_{n}^{2}+2(-1)^{2}+4 P_{n}^{2}+P_{n-1}\left(2 P_{n}+P_{n-1}\right)\right)=P_{n}\left(8 P_{n}^{2}+3(-1)^{n}\right) .
\end{aligned}
$$

Application of the same relations also proves identities for $P_{5 n}$.

Furthermore, for the next instance when $n$ is odd we have

$$
P_{7 n}=512 P_{n}{ }^{7}-448 P_{n}{ }^{5}+112 P_{n}{ }^{3}-7 P_{n}
$$

while all coefficients are positive when $n$ is even.

Theorem 1. For the Pell sequence $\left(P_{n}\right)_{n \geq 0}$

$$
P_{(2 m+1) n}=\sum_{i=0}^{m}(-1)^{n(m+i)} 2^{3 i} \frac{2 m+1}{2 i+1}\left(\begin{array}{c}
m+i \\
2 i
\end{array}\right) P_{n}^{2 i+1} .
$$

Proof. We use equalities (2.5) and (2.6), which are results of D. Jennings available in [9] and which can be proved by induction.

$$
\begin{gathered}
\left(x^{2 m}+\frac{1}{x^{2 m}}\right)+\left(x^{2 m-2}+\frac{1}{x^{2 m-2}}\right)+\cdots+\left(x^{2}+\frac{1}{x^{2}}\right)+1 \\
=\sum_{i=0}^{m} \frac{2 m+1}{m+i+1}\left(\begin{array}{c}
m+i+1 \\
2 i+1
\end{array}\right)\left(x-\frac{1}{x}\right)^{2 i} \\
\left(x^{2 m}+\frac{1}{x^{2 m}}\right)-\left(x^{2 m-2}+\frac{1}{x^{2 m-2}}\right)+\cdots+(-1)^{m+1}\left(x^{2}+\frac{1}{x^{2}}\right)+(-1)^{m} \\
=\sum_{i=0}^{m}(-1)^{m+i} \frac{2 m+1}{m+i+1}\left(\begin{array}{c}
m+i+1 \\
2 i+1
\end{array}\right)\left(x+\frac{1}{x}\right)^{2 i}
\end{gathered}
$$

Having in mind Binet formula for the Pell numbers (1.3) and the fact that

$$
\begin{gathered}
\gamma \cdot \delta=-1 \\
\gamma-\delta=2 \sqrt{2}
\end{gathered}
$$

we have

$$
\frac{P_{p n}}{P_{n}}=\frac{\gamma^{p n}-\delta^{p n}}{\gamma^{n}-\delta^{n}}=x^{p-1}+x^{p-2} y+\cdots+x y^{p-2}+y^{p-1},
$$


where $x=\gamma^{n}$ and $y=\delta^{n}=\frac{(-1)^{n}}{x}$. When $p$ is odd, the r.h.s. of (2.9) reduces to

$$
\left(x^{p-1}+\frac{1}{x^{p-1}}\right)+(-1)^{n}\left(x^{p-1}+\frac{1}{x^{p-1}}\right)+\cdots+\left(x^{p-1}+\frac{1}{x^{p-1}}\right)+(-1)^{n}
$$

when $p \equiv 3(\bmod 4)$ or to

$$
\left(x^{p-1}+\frac{1}{x^{p-1}}\right)+(-1)^{n}\left(x^{p-1}+\frac{1}{x^{p-1}}\right)+\cdots+(-1)^{n}\left(x^{p-1}+\frac{1}{x^{p-1}}\right)+1
$$

when $p \equiv 1(\bmod 4)$. Now, we have

$$
x+\frac{1}{x}=\gamma^{n}+\frac{1}{\gamma^{n}}=\gamma^{n}+(-1)^{n} \delta^{n}
$$

which gives

$$
\begin{aligned}
& x+\frac{1}{x}=(\gamma-\delta) P_{n}, n \equiv 1 \quad(\bmod 2) \\
& x-\frac{1}{x}=(\gamma-\delta) P_{n}, n \equiv 0 \quad(\bmod 2)
\end{aligned}
$$

and furthermore

$$
\begin{aligned}
& \left(x+\frac{1}{x}\right)^{2}=8 P_{n}^{2}, n \equiv 1 \quad(\bmod 2) \\
& \left(x-\frac{1}{x}\right)^{2}=8 P_{n}^{2}, n \equiv 0 \quad(\bmod 2)
\end{aligned}
$$

Since we get expression (2.10) assuming that $p$ is odd we now substitute $p=2 m+1$. Now, when $n$ is even we obtain all positive terms in (2.10) and then r.h.s. of (2.9) is equal to the 1.h.s. of equality (2.5),

$$
P_{(2 m+1) n}=\sum_{i=0}^{m}(-1)^{n(m+i)} 2^{3 i} \frac{2 m+1}{m+i+1}\left(\begin{array}{c}
m+i+1 \\
2 i+1
\end{array}\right) P_{n}^{2 i+1} .
$$

Analogue reasoning when $n$ is odd gives the same relation, thus (2.16) holds true for any natural number $n$. Finally, a simple manipulation with (2.16) leads to the final form of the theorem.

One can easily see that relations (2.1), (2.2) and (2.3) appear from Theorem 1 for $m=1,2$ and 3 , respectively. When $m=4$ Theorem 1 gives

$$
P_{9 n}=2^{12} P_{n}{ }^{9}-9 \cdot 2^{9} P_{n}{ }^{7}+1728 P_{n}{ }^{5}-240 P_{n}{ }^{3}+9 P_{n}
$$

when $n$ is odd while all coefficients are positive otherwise. Note that the leading coefficient in (2.4) is always a power of $2,2^{3 m}$, while the absolute value of the coefficient in the term of the smallest degree is $2 m+1$. 


\section{Melham sum for the Pell and Pell-Lucas Sequence}

Proposition 2. Twice the sum of the Pell numbers having even indexes from 2 to $n$ is equal to the $(2 n+1)$-st Pell number diminished by 1 ,

$$
1+2 \sum_{k=1}^{n} P_{2 k}=P_{2 n+1} .
$$

Proof. The statement follows immediately from defining properties of Pell sequence,

$$
\begin{aligned}
P_{2 n+1} & =2 P_{2 n}+P_{2 n-1} \\
& =2 P_{2 n}+2 P_{2 n-2}+P_{2 n-3} \\
& =2 P_{2 n}+2 P_{2 n-2}+\cdots+2 P_{2}+P_{1} .
\end{aligned}
$$

Note that relation (3.1) can be seen as the expansion of the expression $Q_{1} \sum_{k=1}^{n} P_{2 k}$ into polynomial in $P_{2 n+1}$,

$$
Q_{1} \sum_{k=1}^{n} P_{2 k}=P_{2 n+1}-1 .
$$

In what follows we extend this idea to full generality. The expression

$$
Q_{1} Q_{2} \cdots Q_{2 m+1} \sum_{k=1}^{n} P_{2 k}^{2 m+1},
$$

we shall call the Melham sum for Pell and Pell-Lucas sequences, because there is analogy with established term for Fibonacci and Lucas sequences. More on the Fibonacci sequence one can find in the classic book by S. Vajda. Introduction to Fibonacci polynomials one can find in [8], and some recent development in [2].

Lemma 1. For the sequences $\left(P_{n}\right)_{n \geq 0},\left(Q_{n}\right)_{n \geq 0}$ and $m \in \mathbb{N}$

$$
Q_{m} \sum_{k=1}^{n} P_{2 m k}=P_{m(2 n+1)}-P_{m} \text {. }
$$

Proof. By relation (1.6) we have

$$
\begin{aligned}
P_{m+n} & =\left(P_{m-n}-P_{n} P_{n-1}(-1)^{n}\right)(-1)^{n+1}+P_{m} P_{n-1} \\
& =P_{m-1}(-1)^{n+1}+P_{m} P_{n-1}+P_{m} P_{n+1} \\
& =P_{m-1}(-1)^{n+1}+P_{m}\left(P_{n-1}+P_{n+1}\right) \\
& =P_{m} Q_{n}-(-1)^{n} P_{m-n} .
\end{aligned}
$$


Now we prove the statement of lemma by induction where this result is used in a step of induction. Thus, from the fact that the statement holds true for $n=1$ we have to derive equality $Q_{n} \sum_{k=1}^{n+1} P_{2 m k}=P_{m(2 n+3)}-P_{m}$. We have

$$
\begin{aligned}
Q_{n} \sum_{k=1}^{n+1} P_{2 m k} & =Q_{n}\left(\sum_{k=1}^{n} P_{2 m k}+P_{2 m(n+1)}\right) \\
& =P_{m(2 n+1)}-P_{m}+Q_{n} P_{2 m(n+1)} \\
& =P_{m(2 n+1)}+Q P_{2 m(n+1)}-P_{m} \\
& =P_{2 m(n+1)+m}-P_{m} \\
& =P_{m(2 n+3)}-P_{m},
\end{aligned}
$$

which completes the statement of the lemma.

Lemma 2. For the sequences $\left(P_{n}\right)_{n \geq 0},\left(Q_{n}\right)_{n \geq 0}$ and $m \in \mathbb{N}$

$$
P_{n}{ }^{2 m+1}=\frac{1}{2^{3 m}} \sum_{j=0}^{m}(-1)^{j(n+1)}\left(\begin{array}{c}
2 m+1 \\
j
\end{array}\right) P_{(2 m+1-2 j) n} .
$$

Proof. By means of binomial theorem and using (2.7) as well as (2.8) we have

$$
\begin{aligned}
P_{n}^{2 m+1} & =\left(\frac{\gamma^{n}-\delta^{n}}{\gamma-\delta}\right)^{2 m+1} \\
& =\frac{1}{(\gamma-\delta)^{2 m+1}} \sum_{j=0}^{2 m+1}(-1)^{j+1}\left(\begin{array}{c}
2 m+1 \\
j
\end{array}\right) \gamma^{j n} \delta^{(2 m+1-j) n} \\
& =\frac{1}{8^{m}(\gamma-\delta)} \sum_{j=0}^{m}(-1)^{j}\left(\begin{array}{c}
2 m+1 \\
j
\end{array}\right)\left(\gamma^{(2 m+1-j) n} \delta^{j n}-\gamma^{j n} \delta^{(2 m+1-j) n}\right) \\
& =\frac{1}{2^{3 m}} \sum_{j=0}^{m}(-1)^{j}\left(\begin{array}{c}
2 m+1 \\
j
\end{array}\right) \gamma^{j n} \delta^{j n}\left(\frac{\gamma^{(2 m+1-2 j) n}-\delta^{(2 m+1-2 j) n}}{\gamma-\delta}\right) \\
& =\frac{1}{2^{3 m}} \sum_{j=0}^{m}(-1)^{j(n+m)} P_{(2 m+1-2 j) n}
\end{aligned}
$$

which completes the statement of lemma.

Theorem 2. For $m \in \mathbb{N}$ and the sequences $\left(P_{n}\right)_{n \geq 0},\left(Q_{n}\right)_{n \geq 0}$

$$
\sum_{k=1}^{n} P_{2 k}{ }^{2 m+1}=\frac{1}{2^{3 m}} \sum_{j=0}^{m} \frac{(-1)^{j}}{Q_{2 m+1-2 j}}\left(\begin{array}{c}
2 m+1 \\
j
\end{array}\right)\left(P_{(2 m+1-2 j)(2 n+1)}-P_{2 m+1-2 j}\right) .
$$


Proof. In Lemma 2 we substitute $n=2 k$ and then sum both sides of equality from $k=1$ through $n$. It follows

$$
P_{2 k}^{2 m+1}=\frac{1}{2^{3 m}} \sum_{j=0}^{m}(-1)^{j}\left(\begin{array}{c}
2 m+1 \\
j
\end{array}\right) \sum_{k=1}^{n} P_{(2 m+1-2 j) 2 k} .
$$

When we substitute $\sum_{k=1}^{n} P_{(2 m+1-2 j) 2 k}$ by the expression in Lemma 1, the proof is completed.

Theorem 3. For $m \in \mathbb{N}$ and sequences $\left(P_{n}\right)_{n \geq 0},\left(Q_{n}\right)_{n \geq 0}$

$$
\begin{aligned}
\sum_{k=1}^{n} P_{2 k}^{2 m+1}= & \sum_{i=0}^{m} P_{2 n+1}^{2 i+1} \sum_{j=0}^{m-i} \frac{(-1)^{m+i} 2^{3(i-m)}(2 m-2 j+1)}{Q_{2 m+1-2 j}(2 i+1)}\left(\begin{array}{c}
2 m+1 \\
j
\end{array}\right)\left(\begin{array}{c}
m-j+i \\
2 i
\end{array}\right) \\
& +\sum_{j=0}^{m} \frac{(-1)^{j+1} P_{2 m+1-2 j}}{2^{3 m} Q_{2 m+1-2 j}}\left(\begin{array}{c}
2 m+1 \\
j
\end{array}\right) .
\end{aligned}
$$

Proof. When substitute $m$ with $m-j$ and $n$ with $2 n+1$ in Theorem 1 one get

$P_{(2 m+1-2 j)(2 n+1)}=\sum_{i=0}^{m-j}(-1)^{(2 n+1)(m-j+i)} 2^{3 i} \frac{2 m-2 j+1}{2 i+1}\left(\begin{array}{c}m-j+i \\ 2 i\end{array}\right) P_{2 n+1}^{2 i+1}$.

We substitute this expression in Theorem 2 and the statement follows immediately.

Now we consider some particular cases of Theorem 3. When $m=1$ we obtain

$$
\sum_{k=1}^{n} P_{2 k}^{3}=\frac{1}{14}\left(P_{2 n+1}^{3}-3 P_{2 n+1}+2\right)
$$

When multiply this relation with $Q_{1} Q_{3}$ we get polynomial identity for the Melham sum in case $m=1$

$$
Q_{1} Q_{3} \sum_{k=1}^{n} P_{2 k}^{3}=2 P_{2 n+1}^{3}-6 P_{2 n+1}+4
$$

The next case, when $m=2$ gives

$$
Q_{1} Q_{3} Q_{5} \sum_{k=1}^{n} P_{2 k}^{5}=28 P_{2 n+1}^{5}-120 P_{2 n+1}^{3}+220 P_{2 n+1}-128 \text {. }
$$

\section{FURTHER EXTENSIONS}

Given $s, t \in \mathbb{N}$ and $n \in \mathbb{N}_{0}$ we define the second order recurrence with the relation

$$
a_{n+2}=s a_{n-1}+t a_{n}
$$

and initial values $a_{0}$ and $a_{1}$. We say that a sequence $\left(a_{n}\right)_{n \geq 0}$ is a solution of (4.1) if its terms satisfies this recurrence. Here we consider a class of (4.1) defined by $t=1$ 
and initial terms $a_{0}=0, a_{1}=1$. We let $\left(A_{n}\right)_{n \geq 0}$ denote the sequence defined by this class. It is worth mentioning that two notable representatives of this class are Fibonacci and Pell numbers.

Proposition 3. For the sequence of numbers $\left(A_{n}\right)_{n \geq 0}$ we have

$$
\begin{aligned}
\text { i) } & A_{3 n}=\left(s^{2}+4\right) A_{n}^{3}+3(-1)^{n} A_{n} \\
\text { ii) } & A_{5 n}=\left(s^{2}+4\right)^{2} A_{n}^{5}+5\left(s^{2}+4\right)(-1)^{n} A_{n}^{3}+5 A_{n} .
\end{aligned}
$$

Proof. By induction we prove that

$$
A_{n-1} A_{n+1}-A^{2}=(-1)^{2}
$$

and also

$$
A_{m+n}=A_{m-1} A_{n}+A_{m} A_{n+1} .
$$

Now we employ (4.5) to get

$$
\begin{aligned}
A_{3 n} & =A_{2 n+n}=A_{2 n-1} A_{n}+A_{2 n} A_{n+1} \\
& =A_{n-1}^{2} A_{n}+A_{n}^{3}+\left(A_{n-1} A_{n}+A_{n} A_{n+1}\right)\left(A_{2} A_{n}+A_{n-1}\right) \\
& =A_{n}\left(A_{n-1}^{2}+A_{n}^{2}+s A_{n-1} A_{n}+s A_{n} A_{n+1}+A_{n-1}^{2}+A_{n-1} A_{n+1}\right) .
\end{aligned}
$$

Having in mind that

$$
A_{n-1}^{2}+s A_{n-1} A_{n}=A_{n}^{2}+(-1)^{n}
$$

by (4.4), we obtain

$$
A_{3 n}=A_{n}\left(2 A_{n}^{2}+2(-1)^{n}+A_{n}^{2}+s A_{n} A_{n+1}+A_{n-1}^{2}\right) .
$$

When applying again (4.4) to the terms $s A_{n} A_{n+1}$ and $A_{n-1}^{2}$ we finally have

$$
\begin{aligned}
A_{3 n} & =A_{n}\left[4 A_{n}^{2}+s^{2} A_{n}^{2}+3(-1)^{n}\right] \\
& =A_{n}\left[\left(s^{2}+4\right) A_{n}^{2}+3(-1)^{n}\right] .
\end{aligned}
$$

The second relation can be proved by analogue calculation.

Clearly, further identities can be proved in the same fashion as Proposition 3 was proved. Instead, we give a more elegant family of identities (4.6) that generalize Proposition 3. It follows as a corollary of Theorem 1.

Corollary 1. For $m \in \mathbb{N}$ and the sequence of numbers $\left(A_{n}\right)_{n \geq 0}$ we have

$$
A_{(2 m+1) n}=\sum_{i=0}^{m}(-1)^{n(m+i)}\left(s^{2}+4\right)^{i} \frac{2 m+1}{2 i+1}\left(\begin{array}{c}
m+i \\
2 i
\end{array}\right) A_{n}^{2 i+1} \text {. }
$$


In order to prove Corollary 1 we use the fact that the closed form relation for the terms of sequence $\left(A_{n}\right)_{n \geq 0}$ is

$$
A_{n}=\frac{\alpha^{n}-\beta^{n}}{\alpha-\beta},
$$

where

$$
\alpha=\frac{1}{2}\left(s+\sqrt{s^{2}+4}\right), \quad \beta=\frac{1}{2}\left(s-\sqrt{s^{2}+4}\right) .
$$

Furthermore, it holds $\alpha \cdot \beta=-1, \alpha-\beta=\sqrt{s^{2}+4}$ which generalize relations (2.7) and (2.8) in the proof of Theorem 1. This completes the statement of Corollary 1.

Further generalizations and extensions of expressions presented in this work are also possible.

\section{ACKNOWLEDGEMENT}

The authors are thankful to Professor B. Sury from the Indian Statistical Institute, Bangalore for providing useful refrerence.

\section{REFERENCES}

[1] J. Aguirre, A. Dujella, and J. C. Peral, "Arithmetic progressions and Pellian equations.” Publ. Math., vol. 83, no. 4, pp. 683-695, 2013, doi: 10.5486/PMD.2013.5684.

[2] T. Amdeberhan, X. Chen, V. H. Moll, and B. E. Sagan, "Generalized Fibonacci polynomials and fibonomial coefficients." Ann. Comb., vol. 18, no. 4, pp. 541-562, 2014, doi: 10.1007/s00026014-0242-9.

[3] J. Beck, "Pell equation and randomness." Period. Math. Hung., vol. 70, no. 1, pp. 1-108, 2015, doi: 10.1007/s10998-014-0064-x.

[4] A. T. Benjamin, S. S. Plott, and J. A. Sellers, "Tiling proofs of recent sum identities involving Pell numbers." Ann. Comb., vol. 12, no. 3, pp. 271-278, 2008, doi: 10.1007/s00026-008-0350-5.

[5] J. J. Bravo, P. Das, S. Guzmán, and S. Laishram, "Powers in products of terms of Pell's and Pell-Lucas sequences." Int. J. Number Theory, vol. 11, no. 4, pp. 1259-1274, 2015, doi: $10.1142 / \mathrm{S} 1793042115500682$.

[6] A. Dujella, "A problem of Diophantus and Pell numbers." in Applications of Fibonacci numbers. Volume 7: Proceedings of the 7th international research conference on Fibonacci numbers and their applications, Graz, Austria, July 15-19, 1996. Dordrecht: Kluwer Academic Publishers, 1998, pp. 61-68.

[7] S. Falcón Santana and J. L. Díaz-Barrero, "Some properties of sums involving Pell numbers." Missouri J. Math. Sci., vol. 18, no. 1, pp. 33-40, 2006.

[8] R. L. Graham, D. E. Knuth, and O. Patashnik, Concrete mathematics: a foundation for computer science. 2nd ed., 2nd ed. Amsterdam: Addison-Wesley Publishing Group, 1994.

[9] D. Jennings, "Some polynomial identities for the Fibonacci and Lucas numbers." Fibonacci Q., vol. 31, no. 2, pp. 134-137, 1993.

[10] E. Kiliç, N. se Ömür, and Y. T. Ulutas, "Alternating sums of the powers of Fibonacci and Lucas numbers.” Miskolc Math. Notes, vol. 12, no. 1, pp. 87-103, 2011.

[11] M. Werman and D. Zeilberger, "A bijective proof of Cassini's Fibonacci identity." Discrete Math., vol. 58, p. 109, 1986, doi: 10.1016/0012-365X(86)90194-9. 
[12] A. Wloch and M. Wolowiec-Musial, "Generalized Pell numbers and some relations with Fibonacci numbers.” Ars Comb., vol. 109, pp. 391-403, 2013.

Authors' addresses

I. Martinjak

University of Zagreb, Faculty of Science, Bijenička 32, 10000 Zagreb, Croatia

E-mail address: imartinjak@phy.hr

I. Vrsaljko

University of Zagreb, Faculty of Science, Bijenička 32, 10000 Zagreb, Croatia

E-mail address: iva.vrsaljko91@gmail.com 